\title{
A Study on Pricing and Emission Reduction Decision of Steel Supply Chain under Free Carbon Quota Policy
}

\author{
Yanling $\mathrm{Wu}^{\mathrm{a}}{\text {, Zhigao } \text { Liao }^{\mathrm{b}} \text { and } \mathrm{Yu} \text { Chen }}^{\mathrm{c}}$ \\ School of Management, Guangxi University of Science \& Technology, Liuzhou 545006, China. \\ awyl521510@163.com, b46154910@qq.com, ccy31600@163.com
}

Keywords: Steel supply chain, pricing decision, emission reduction decision, historical method, benchmark method.

\begin{abstract}
Considering that the retailer bears part of the manufacturer's emission reduction cost, this paper analyzes the optimal pricing and optimal emission reduction decisions for the two-level steel supply chain under the carbon-free policy, historical method and benchmark method. The numerical example shows that the emission reduction cost sharing rate is positively related to the manufacturer's emission reduction rate. Compared with the historical method, the benchmark method can effectively promote the manufacturers to reduce carbon emission. Under the benchmark method, when the retailer bears the appropriate emission reduction cost for manufacturers, it can promote the two-way development of the steel supply chain economy and emission reduction.
\end{abstract}

\section{Introduction}

The emission of greenhouse gases leads to global warming, which has become an important issue for the sustainable development of the global economy. As a pillar industry of China's national economy, the steel industry accounts for about $15 \%$ of the country's total carbon emissions. The steel industry is one of the emission reduction industries, and China has not proposed a clear carbon quota allocation method. At present, the national carbon trading market is in the prototype stage. Most carbon trading pilots use free distribution, and there are mainly historical method and benchmark method for free distribution. Therefore, adopting the historical method or the benchmark method has become a practical issue in the steel industry. In addition, the issue of supply chain operation decision-making under the carbon trading policy has also gradually received the attention of some scholars. In terms of pricing and production decision-making, $\mathrm{Xu}$ et al discussed the issue of joint production and pricing of manufacturers under cap-and-trade and carbon tax policies[1]; Jialing Jiao constructed two kinds of game models about the enterprise to join the carbon trading system and accept the extra punishment without joining, and the effects of different emission quotas, transaction prices, and penalties on the pricing decisions of the enterprise were explored through numerical simulation [2]. In terms of inventory management and order quantity, Song et al used the newsboy model to analyze the issue of single-cycle optimal order quantity decision-making under the three kinds of carbon emission policies under random demand [3]; In the two-stage supply chain system composed of suppliers and purchasers, Wei Zeng solved the optimal order quantity of enterprises under the carbon trading mechanism through economic batch model [4]. In addition to the above researches, some scholars have also conducted research on emission reduction decisions. Yourdon $\mathrm{Li}$ and Daisha Zhao constructed two game forms, Nash game and Stackelberg game, which were made up of manufacturers and retailers, and obtained government subsidies and strategy for cooperative emission reduction of supply chain companies under the different forms of game [5].

To sum up, the research on the impact of carbon trading on supply chain operations has mainly focused on the aspects of pricing, production, inventory management, and optimal order quantity, etc. There is relatively little research on carbon emission reduction. In the few literatures on carbon emission reduction, few literatures have conducted in-depth research on carbon trading policies. Based on this, this article analyzes the pricing and emission reduction decisions of the steel supply 
chain under different free carbon quota policies, taking into account the retailer's sharing of the manufacturer's emission reduction costs.

\section{Problem Description and Model Hypothesis}

At present, there are many uncontrolled companies that are not subject to carbon trading policies in the steel supply chain, which is not conducive to the overall emission reduction of the steel supply chain. In view of this, this paper constructs a two-level supply chain consisting of a steel manufacturer and a steel retailer. The steel manufacturer is the control company, the steel retailer is the non-control company, and the country considers the supply chain as a whole and supply chain carbon quota to the manufacturer. The manufacturer carries out carbon emission reductions under the constraints of carbon trading policies. At the same time, it constrains the retailer through product pricing, and the retailer bears some carbon emission reduction costs of the manufacturer. After both sides of the game to achieve the goal of common emission reduction.

This article has the following hypothesis.

Hypothesis 1: According to the assumption of the classical model standard, the manufacturer's carbon emission reduction cost has a secondary relationship with its emission reduction rate, $C\left(\tau_{i}\right)=1 / 2 \beta_{i}\left(\tau_{i}\right)^{2}, \beta_{i}$ is emission reduction investment cost factor, and $\beta_{i}>0,0<\tau_{i}<1$ [6].

Hypothesis 2: As consumers' low-carbon awareness increases, consumer demand meets $Q=a-b p_{r}+c \tau_{m}, a$ is the original market demand of the product, $b$ is the impact factor of price on market demand, and $c$ is the sensitivity of consumers to carbon emissions. And $a>0, b>0$, $a>b p_{r}, c>0[7]$.

The main model parameters of this article are as follows: $p_{m}$ and $p_{r}$ refer to the unit wholesale price and retail price; $c_{m}$ and $c_{r}$ refer to the total cost per unit of manufacturer, retailer; $\tau_{m}$ refers to the emission reduction rate; $Q$ refers to the demand; $e_{m}$ refers to the carbon emissions from unit products before reduction; $\xi_{m}$ refers to the carbon quotas allocated according to total amount; $\alpha_{m}$ refers to the carbon quotas allocated per unit of production; $p_{e}$ refers to the unit carbon trading price; $\lambda$ refers to the emission reduction cost sharing rate; $\pi_{m}, \pi_{r}$ and $\pi$ refer to the profits of the manufacturer, the retailers and supply chain.

\section{The Establishment and Solution of the Model}

\subsection{Carbon-Free Policy Game Model.}

Without the carbon policy, the manufacturer's profits consist of sales revenue and carbon emission reduction costs, the retailer's profits consist of sales revenue and share of the manufacturer's carbon emission reduction costs. The manufacturer prioritizes the decision on the wholesale price and the emission reduction rate, then the retailer determines the retail price. The game model is as follows:

$$
\begin{aligned}
& \pi_{\mathrm{m} 1}=\left(p_{m}-c_{m}\right) Q-\frac{1}{2}(1-\lambda) \beta_{m} \tau_{m}{ }^{2} \\
& \pi_{\mathrm{r} 1}=\left(p_{r}-p_{m}-c_{r}\right) Q-\frac{1}{2} \lambda \beta_{m} \tau_{m}{ }^{2}
\end{aligned}
$$

\subsection{Game Model Based on History Method.}

The historical method refers to the method of determining the carbon emission quota for future years by using the carbon emissions data of units that have been included in the quota management in the previous year as the main basis. Under this policy, the game model is as follows:

$$
\begin{aligned}
& \pi_{m 2}=\left(p_{m}-c_{m}\right) Q-\left[e_{m}\left(1-\tau_{m}\right) Q-\xi_{m}\right] p_{e}-\frac{1}{2}(1-\lambda) \beta_{m} \tau_{m}{ }^{2} \\
& \pi_{r 2}=\left(p_{r}-p_{m}-c_{r}\right) Q-\frac{1}{2} \lambda \beta_{m} \tau_{m}{ }^{2}
\end{aligned}
$$




\subsection{Game Model Based on the Benchmark Method.}

The benchmark method is to set a benchmark first, and then determine the allocation of quota based on the product of the actual emissions of the regulated companies and the benchmark. This game model is as follows:

$$
\begin{gathered}
\pi_{m 3}=\left(p_{m}-c_{m}\right) Q-\left[e_{m}\left(1-\tau_{m}\right)-\alpha_{m}\right] Q p_{e}-\frac{1}{2}(1-\lambda) \beta_{m} \tau_{m}{ }^{2} \\
\pi_{r 3}=\left(p_{r}-p_{m}-c_{r}\right) Q-\frac{1}{2} \lambda \beta_{m} \tau_{m}{ }^{2}
\end{gathered}
$$

\subsection{Game Result Analysis.}

Proposition 1: Under historical method, let $A=a-b\left(c_{m}+c_{r}\right), B=c+b e_{m} p_{e}$, if $4 b \beta_{m}(1-\lambda)-B^{2}>0$, there was a unique optimal solution for corporate decisions in the supply chain:

$$
\left\{\begin{array}{c}
p_{m 2}=\frac{2 \beta_{m}(1-\lambda)\left[a+b\left(c_{m}-c_{r}+e_{m} p_{e}\right)\right]-B\left[c \cdot c_{m}+\left(a+c-b c_{r}\right) e_{m} p_{e}\right]}{4 b \beta_{m}(1-\lambda)-B^{2}} \\
\tau_{m 2}=\frac{B\left(A-b e_{m} p_{e}\right)}{4 b \beta_{m}(1-\lambda)-B^{2}} \\
p_{r 2}=\frac{(1-\lambda) \beta_{m}\left[3 a+b\left(c_{m}+c_{r}+e_{m} p_{e}\right)\right]-B\left[c\left(c_{m}+c_{r}\right)+(a+c) e_{m} p_{e}\right]}{4 b \beta_{m}(1-\lambda)-B^{2}}
\end{array}\right.
$$

Proof: Using the inverse induction method to solve, formula (4) finds the first derivative of $p_{r}$, makes it equal to zero, brings the value of the solution into $Q$, then $Q$ into formula (3), and it takes the partial derivative with respect to $p_{m}$ add $\tau_{m}$. Let it be equal to zero. When $4 b \beta_{m}(1-\lambda)-B^{2}>0$, we get: $p_{m 2}$ and $\tau_{m 2}$. At this time, the Hasselblad matrix of formula (3) is negative definite, so $p_{m 2}$ and $\tau_{m 2}$ are the optimal solutions of formula (3).Substituting $p_{m 2}$ and $\tau_{m 2}$ into $p_{r}$ gives $p_{r 2}$. This $p_{r 2}$ is the optimal solution of (4), and Proposition 1 is proved.

Proposition 2: There are the following relationships between the emission reduction rates under the two carbon policies: $\tau_{m 2}<\tau_{m 3}$

Proof: $\tau_{m 3}-\tau_{m 2}=\frac{b \alpha_{m} p_{e}\left(c+b e_{m} p_{e}\right)}{4 b(1-\lambda) \beta_{m}-\left(c+b e_{m} p_{e}\right)^{2}}>0$, it can be seen that the emission reduction rate under the benchmark method is always greater than the emission reduction rate under the historical method, which means that the benchmark method can promote the emission reduction of manufacturers more than the historical method.

\section{The Example Analysis}

In order to analyze the influence of the emission reduction cost sharing rate on the supply chain decision variables in the above three models, this section uses a numerical analysis method to perform the correlation analysis. Under the condition of satisfying the optimal solution, let $a=100$, $b=1.25, \beta_{m}=200, p_{e}=1, c_{m}=15, c_{r}=3, e_{m}=3, \xi_{m}=60, \alpha_{m}=2, c=2$.Figure 1 to figure 6 respectively represent the impact of emission reduction rate on emission reduction rate, wholesale price, retail price, manufacturer profit, retailer profit and total supply chain profit. 


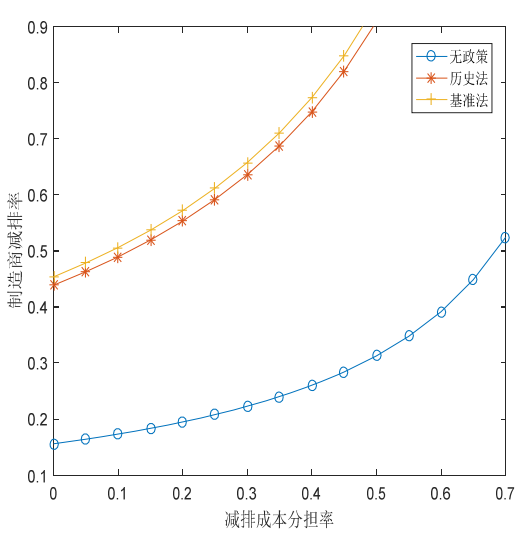

Fig. 1. Effect of $\lambda$ on $\tau_{m}$

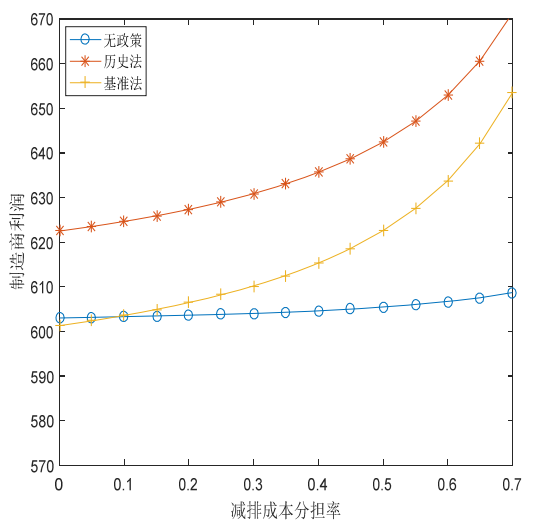

Fig. 4. Effect of $\lambda$ on $\pi_{m}$

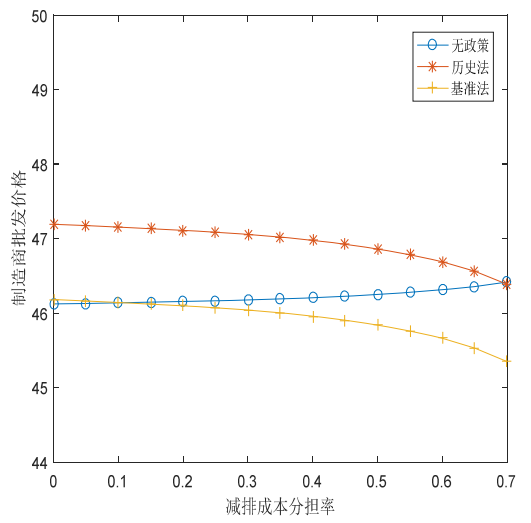

Fig. 2. Effect of $\lambda$ on $p_{r}$

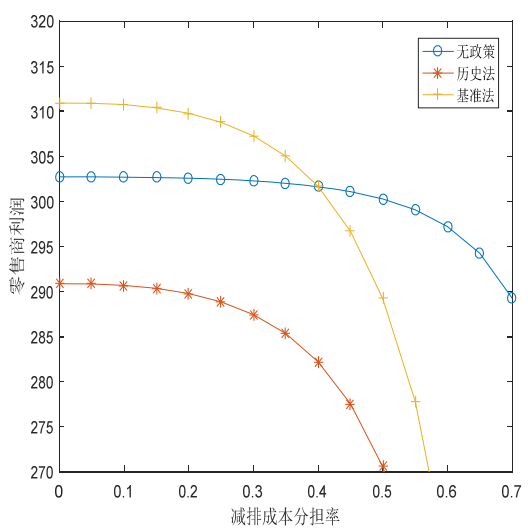

Fig. 5. Effect of $\lambda$ on $\pi_{r}$

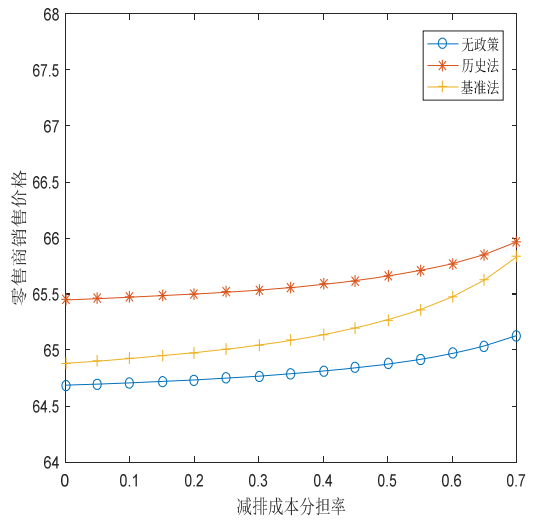

Fig. 3. Effect of $\lambda$ on $p_{m}$

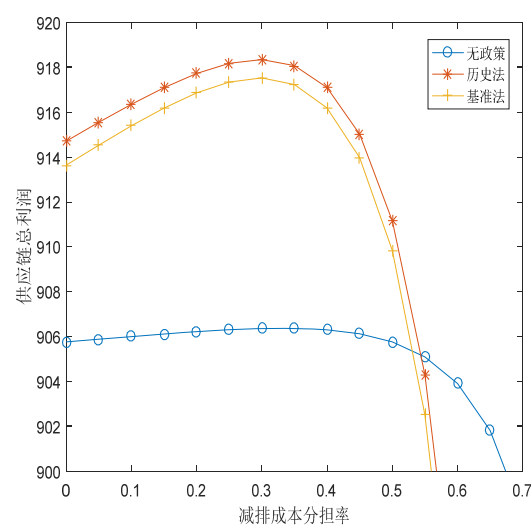

Fig. 6. Effect of $\lambda$ on $\pi$

Through numerical analysis, it is found that data with $\lambda$ greater than 0.7 is subject to greater fluctuations. Considering the fact that retailers cannot afford more than $70 \%$ of $\lambda$, the range of $\lambda$ for this study is between 0 and 0.7. From Figure 1 to Figure 6, it can be seen that in addition to Figure 2 and Figure 5, the decision variable images under the non-policy are basically under two kinds of carbon policies, which shows that the implementation of carbon policies is beneficial to supply companies.

Figure 1 shows that $\tau_{m 1}<\tau_{m 2}<\tau_{m 3}$, verifying Proposition 2 . In addition, $\tau_{m}$ increases with the increase of $\lambda$, which means that the retailer's sharing of emission reduction costs for manufacturers can promote carbon emission reduction by manufacturers. In Figure 2 and Figure 3, $p_{r}$ under the carbon policy decreases with the increase of $\lambda$, while $p_{m}$ increases with the increase of $\lambda$, which means that the greater the emission reduction cost the retailer bears, the greater the total cost of the retailer, the smaller the total cost of the manufacturer. Figure 4 and figure 5 shows that $\pi_{m}$ increase with the increase of $\lambda$, and $\pi_{r}$ are reduced with the increase of $\lambda$, indicating that the increase of $\lambda$ enables manufacturers to reduce costs and improve profits, but it increases the retailer's cost and reduces the profit. Figure 6 shows that the graph under the carbon policy first rises and then falls, indicating that $\lambda$ is not the bigger the better. When $\lambda=0.3$, the total profits of the supply chain under the carbon policy have reached the maximum value and are much greater than the total profits without policies.

\section{Conclusion}

This paper constructs a two-level Steinberg game model to study the optimal decision-making of supply chain members under the carbon-free policy, historical method and benchmark method. The study found that with the historical method, the benchmark method is more effective in promoting carbon reduction by steel manufacturers. The benchmark method is more suitable for the development of the steel supply chain, because under the benchmark law, when $\lambda=0.3$, emission 
reductions are maximized, and profits of manufacturers and retailers are greater than those of carbon-free policies. Under the historical law, the profit of the manufacturer is greater than that of the carbon-free policy, while the retailer's profit is less than the profit when the carbon-free policy is adopted. Therefore, for the steel supply chain, under the benchmark method, when the retailer bears the appropriate emission reduction cost for the manufacturer, it can not only promote low-carbon emission reduction in the steel supply chain, but also promote the economic development of the steel supply chain.

\section{Acknowledgments}

We acknowledge the support of Guangxi Philosophy and Social Sciences Research Topics” Effects of Carbon Trading on Guangxi's Manufacturing Carbon Emissions and Countermeasures” (17FJY007).

\section{References}

[1]. Xu Xiao an, Xu Xiaoping, He Ping. Joint production and pricing decisions for multiple products with cap-and-trade and carbon tax regulations [J]. Journal of Cleaner Production, 2016,112 (20):4093 -4106.

[2]. Jiao Jialing, Chen Jibe, Li Lankan, Li Fungi. Evolutionary Game Analysis of Behaviors of Local Governments and Enterprises under the Mechanism of Reward and Punishment for Carbon Emission Reduction [J]. Chinese Journal of Management Science, 2017, 25(10):140 150.

[3]. Song J P, Lang M. Analysis of the single-period problem under carbon emissions policies [M]//Choi T M. Handbook of Newsvendor Problems: Models, Extensions and Applications. New York: Springe, 2012:297 313.

[4]. Zeng Wei, Wang Yoshi, Zhou Hangtag. Optimization of Supply Chain Operation under Carbon Limit and Trading Mechanism [J]. Journal of Industrial Engineering and Engineering Management, 2015, 29(03): 199 206.

[5]. Li Yourdon, Zhao Daisha. A Comparative Study of R\&D Cost Allocation in Low-carbon Supply Chains Considering Government Subsidies [J]. Soft Science, 2014, 28(3):21 31.

[6]. Zhang Zhanjiang, Zhang Jay, Lai Mingy Ong. Game Analysis of Cooperation and R\&D of Government Behavior and Supply Chain under Low-carbon Background [J]. Chinese Journal of Management Science, 2015, 23(10):57 66.

[7]. Fan Shining, Wang Qing, and Chen Li. Research on three-level low carbon supply chain emission reduction game under government subsidies [J]. Industrial Technology \& Economy, 2017, 36(11): 12 20. 